\title{
Functionalities provided by Annotation Systems for Learners in Educational Context: An Overview
}

\author{
http://dx.doi.org/10.3991/ijet.v11i02.5166 \\ Anis Kalboussi ${ }^{1,2}$, Omar Mazhoud ${ }^{1,2}$, and Ahmed Hadj Kacem ${ }^{1}$ \\ ${ }^{1}$ Kairouan University, Kairouan, Tunisia \\ ${ }^{2}$ Sfax University, Sfax, Tunisia
}

\begin{abstract}
During his activities, the learner usually uses comments, highlights and circles sections to tag consulted digital documents. Therefore, many systems have been developed to annotate different electronic resources, often adapted to specific users and for particular uses of documents. This great variety of annotation systems reveals a lack of a clear strategy of how to compare the developed annotation systems of the literature according to their functionalities. As a result, few works have tried to present comparative studies of these tools. The aim of this article is to provide a study of some annotation tools used by learners in educational practices. Therefore, we present a comparison of functionalities provided by forty annotation systems developed by industry and academia during the last decade. The study further reveals gaps in systems and opportunities for further research.
\end{abstract}

Index Terms - Survey, annotation system, functionalities, comparative study, learner.

\section{INTRODUCTION}

Annotation systems are widely used by learners in educational practices because annotations give students the chance to summarize new ideas while receiving peer support [12]. Tagging can be considered as an action of reflection, where the learner can recapitulate a series of thoughts into one or more annotations, each of which stands on its own to describe some aspect of the resources based on tagger's experiences and beliefs [3].

Furthermore, annotations are not only a way of explaining and enriching an information resource with personal learner observations, but also a means of transmitting and sharing ideas to improve collaborative work practices [41]. Thus, the possibility of enriching digital contents by adding annotations has attracted many researchers, who have looked at this opportunity from many different perspectives and with a number of purposes in mind [3, 29, 39].

Therefore, several annotation systems have been developed in learning environment adapted for various contexts and for various roles. Researchers have mostly focused on providing more and more sophisticated interfaces with several annotation functionalities. These annotations tools help learners to organize and understand learning materials, and to add a personalized view [33].

However, these annotation systems differ in their functionalities and features. The great variety makes it more difficult to get an overview and to decide which system to choose for a certain learning scenario. As a result, there is only a fragmentary picture of functionalities provided by these annotation tools to learner in educational context. This flourishing of different works about annotation systems reveals a lack of how to compare the developed tools of the literature according to their functionalities.

To compensate for this lack, we propose in this article a comparative study of forty annotation tools used by learners in educational practices. This comparison involves seven main functionalities provided by annotations tools which are synthesized from the state of the art of all studied systems: to manage annotations (create/ delete/ modify); to store annotations; to visualize stored annotations; to search annotations; to share annotations; to notify and answer to annotations and to recommend data related to annotations.

This article is organized as follows: At first, we provide a set of definition of learner's annotations in educational context presented by researchers. Then, we propose a comparative study of some annotation tools used by learners in educational practices depend on functionalities and features of these annotation systems. Finally, based on this panoramic study, we draw some key observations, limitations and a discussion of open research issues.

\section{ANNOTATION IN EDUCATIONAL PRACTICES}

Annotation is an educational practice which bridges between reading and writing and constitutes the most prominent habits of active reading for learner [32]. We mean by annotation the act to add a critical or explanatory note to a consulted resource, to highlight a passage, to write down, etc., that learner makes on a page during reading activity [31].

Marshall [54] defines the annotation as " $a$ tangible demonstration of the commitment of the learner with the text". In the context of computer supported collaborative learning, $\mathrm{Su}$ et al. [17] indicate that "an annotation refers to an explicit expression of knowledge that is attached to a document to reveal the conceptual meanings of an annotator's implicit thoughts". Chen et al. [16] define the annotation as "a strategy for active reading wherein you write the key information in the margins of your text, because it gives you a purpose, you'll find that annotation helps you concentrate while reading, and it actually helps you learn from the text". According to Cabanac et al. [41], annotation is " $a$ track of the mental state of the learner and a track of his reactions towards the document. The latter is characterized by tracks which reflect the interest and the activity of the reader".

From the previous definitions, it seems clear the importance of annotative activity in learning process for learner. Thus, many annotation systems have been 
developed since the $90^{\text {th }}$ to transpose on electronic document the practice of annotation of learner. Then, these systems have gradually taken advantage of processing capabilities and communication of modern computers to enrich the practice of digital annotation [21]. An annotation system or still called annotation tool is a system allowing users to annotate various types of electronic resources with different kind of annotations. Many researchers have been interested in the creation of annotation tools to facilitate learner's annotative activity in digital environment. We can find numerous commercial software and research prototypes created to annotate electronic educational resources such as: TafAnnote [41]; Notebook [26]; Crocodoc [46]; New-WebAnnot [2]; etc.

\section{FunCtionAlities PROVIDED By ANNOTATION TOOLS FOR LEARNER}

Many attempts have been made to build annotation systems in learning environment [3]. These systems take several different approaches to provide learners with the option to annotate various digital resources. Thus, annotation tools implement a wide range of functionalities that often go beyond those available in traditional pen and paper annotation [25]. They offer a huge diversity of annotation types that can be placed in textual as well as in multimedia format [22]. Learner is in the center of annotation systems. Thus, numerous information systems offer functionalities close to paper annotation practices: highlight, underline, attach a note, etc. Besides, these softwares take advantage of capacities of processing of a computerized information system: research, automatic classification, sharing, recommendations, etc. The following sections expose a synthesis of functionalities proposed by studied annotation systems:

\section{A. To manage annotations (create/ delete/ modify)}

The transposition of paper annotative activity on digital environment enables the annotation of a wider range of resources. The supports for the annotation are multiple. By using the expressiveness of the HTML language, advanced text formatting (fonts, colors, underlining, etc.), images, sounds, videos, etc., are possible [47]. In addition, beyond HTML, the advanced capabilities of modern graphical interfaces are used to represent non-textual annotations in electronic letters shapes drawn with the mouse [7]. Yet, Kahan et al. [29] report that it is rare to find an annotation system for performing annotations as fluid as on paper as the use of the mouse in this task is uncomfortable. However, the technology rapidly evolving, Chatti et al. [9] quote the digital ink used to write with a stylet on a touch screen PDA for example. The formulation of handwritten electronic annotations is therefore possible, allowing a high fluidity in shapes [43].

Existing annotation systems come in two categories. The first ones allow associating an annotation to the whole annotated resource (located by its URL). The second are used to associate an annotation to a portion of consulted resource (located by its URL and the annotated passage). In the first case, the anchor of annotation is the whole resource. While, in the second, it is a portion of resource. This choice has significant repercussions on the architecture and features of an annotation system. Once the anchor of the annotation has been defined, the information added by annotator is generally: (Annotating content, context and the semantics of annotation). These data added by learner have no impact on the architecture of annotation system; they are simply attributes that can be filled when creating annotation [51]. To create an annotation, the system must know the URL annotated by learner. A simple solution would be to ask user to give himself the URL of resource which he wants to annotate, and he also meets the other attributes of annotation (type, context, semantic, etc.). This information would then be transmitted to annotation server [23]. In practice, this would be of course painful for learner [8]. Thus, annotation system must provide to student an easy way to add a new annotation. Two categories of solutions were emerged. One based on an intermediate 'Proxy' and the other through an intermediate 'Customer':

- Intermediate 'Proxy' to create an annotation. Before sending back the annotated resource, the intermediary proxy takes care of an ultimate step: it includes HTML buttons at the top of the resource and a JavaScript code [18]. When learner wants to create a new annotation, he clicks on this button directly in the resource and the associated JavaScript code opens a window offering to complete a form [43]. After completing this form, the script sends its content to annotation server.

- Intermediate "Customer" to create an annotation. An intermediary customer has access to the loaded resource. In particular, it can know which part of resource is currently selected, and it is thus straightforward to create a new annotation [15]. Once again, the graphical interface of the intermediary "Customer" is not tied to a particular language (such as JavaScript and HTML in the case of proxies). Furthermore, the original resource does not need to be modified to include annotation buttons there [18]. All elements concerning the annotation can be relegated to the graphical interface of the intermediary, even if this one is visually linked to the browser [47].

Through our literature study, we can meet several procedural modes of creation of annotation. We differentiate the cases where learner selects at first the anchor and then the shape of the annotation to be applied, and the cases where learner chooses at first the shape of annotation and then the anchor in which to apply it. The first procedure is called (object/tool) and the second (tool/object). We distinguish then the accesses by buttons and the accesses by menus. These menus are presented in two formats (drop-down menus) or (gushing menus) [51].

Two strategies are possible for the creation of an annotation relative to a consulted resource. The first one consists on integrating a link towards the annotation into the resource whose content is modified [29]. When we cannot integrate annotations into resources because they are in only reading (for example it is the case on the Web) it is necessary to resort to a technique of anchoring to keep the link annotation-resource without modifying the original resource [6]. A first technique of anchoring consists in memorizing the selected annotated content as well as its rank in the resource. It allows distinguishing it among the possible occurrences of the same passage in the resource. This technique is intuitive, simple to implement, but not very robust because it is little resistant to the modifications brought to the resource [17]. Kahan et al. [29] propose a more robust technique for semi-structured resource, where the anchor point of the selected passage is the path (way) in the logical structure of the resource. This 
type of anchor has been standardized by the $\mathrm{W} 3 \mathrm{C}$ as part of XML documents where the path of an element can be expressed through the XPointer language [42]. This allows to formulate anchor points even within an XML element, providing a granularity finer than XPath. XPointer is specifically used in Annotea [29] to create rather relative anchor points, so that they are less sensitive to modifications upstream in the structure of resource.

\section{B. To store annotations}

Once created by the learner, annotations should be stored in order to exploit them. The selected anchor point as well as the information related to annotation typically (title, author, date, visible indication, etc.) are stored in an annotation server [40]. Many learners using the same server can then share their annotations and visualize those of the other students [20]. WebAnnot [4], CATool [43] IIAF [21] and VSim [19] store annotations in a structured text file into fields. Thus, stored annotations can be shared among multiple learners through this file accessible via the network. Eye-Gaze [5], ASRLM [16], Livenotes [14], and A.nnotate [44] are based on the model 'Common Annotation Framework' expressed in Resource Description Framework (RDF) [55] and interpreted by associated server. Although, the storage of annotations outside of annotated resource is rather complex. It is necessary to define an anchor as robust as possible and merge annotations with resource in the restoration [21]. This approach is very convenient for asynchronous collaboration tasks such as work team and reviews of documents [44]. Centralizing annotations on a dedicated server allows to search annotation among all those who are public, to ask to be notified when comments are added, etc. From a technical view point, when a learner attaches an annotation to a web educational resource, the data describing this annotation are stored in one or more servers [30]. Some annotation systems define groups and access rights to annotations while others do not make it [17]. Finally, the W3C [29] provides access to a public annotation server for purposes of tests. The source code of Annotea server is available; organizations have therefore compiled and installed their own annotation server. According to the creators of Annotea, many aspects of the server, especially the data model, are easily expandable and offer interesting possibilities for future works [29].

\section{To visualize stored annotations}

We observed in the literature two strategies to visualize stored annotations of a resource to the annotator:

- Representation out of context. Contrary to 'pen and paper' annotation practices, some annotation systems do not change at all the original layout of annotated resource. For example, CoScribe [10] and PaperCP [27] post annotations in a frame near to the annotated resource, taking then the metaphor of the margin in a sheet of paper. Other systems such as Crocodoc [46] and EndNotes [8] visualize annotations in a schedule window, without incorporating them into their original resource. This type of visualization requires cognitive effort from learner to mentally merge annotations and the annotated resource. Furthermore, it is difficult to handle both windows (browser and frame). These limitations have led the recent annotation systems to incorporate annotations into the context, even within annotated resource [15].
- $\quad$ Representation in context. It represents the alternative which integrates annotations within original resource. At the opposite of annotations systems that do not change the displayed resource, some tools incorporate all of the annotation. Thus, PDF-Annotator [49] or Microsoft-OneNote [48] highlight annotations called 'inline' by inserting comments into the annotated resource with a style (police and color of annotation) chosen by learner (author) and customizable by reader [50]. This allows to make well the difference between the annotation and the original annotated content. When the contents of an annotation exceed a certain size, a pop-up window will be automatically opened to insert in it the textual annotation [38]. On the other hand, some researchers highlight the difficulty of displaying annotations in the margin of a HTML document. This is why alternative techniques have been developed to resolve this problem. Thus, new annotation systems insert into the annotated resource only an icon which indicates the beginning of the anchor point of the annotation, as for example New-WebAnnot [2], MyNote [6], and WriteOn [13]. Icons also serve as hyperlinks which allow to see the content of annotation by placing the mouse pointer above the icon. The content is displayed in a tooltip. By clicking on the link, the learner can access to additional features: add a comment, copy the contents, see the profile of the annotator, etc. [31].

\section{To search annotations}

By annotating electronic educational resources, learners can benefit from capacities of computing processing. The majority of annotation tools provide the functionality of searching for annotations. Two procedures are possible: the path (very common) or search (rarer). In the first case, the annotator must browse all the annotations, until he found that he looks for [16]. Sometimes he can browse only the annotations of certain shape. In the second case, the annotator looks for a specific annotation by giving some of its characteristics (content, author, date, etc.). The search for annotations by using keywords is an effective research technique: a search engine indexing annotations drastically reduces the time spent looking for a passage [54]. This feature is especially appreciated by learners who delve into piles of paper articles for a particular passage. Despite the utility of such feature, Chen et al., [23] relate that few systems allow actually to search for annotations. Therefore, recent annotation systems try to propose the functionality of searching of annotations with keywords $[2,4]$.

\section{E. To share annotations}

Learners have usually the habit to share electronic resources which they have previously annotated them in many circumstances. As part of university research, exchange of annotated scientific articles is current and common. However, web browsers are designed for solitary use and allow little exchange within a group. Nevertheless, learners increasingly need to share resources with groups of students having the same interests [37]. The need to share educational resources is reflected into annotation systems. Some tools allow to exchange annotations by e-mail or instant messaging software as ICQ8 for We-LCoME [28]. From the proposal of W3C recommendation for the RDF-schema, designers of annotation systems intend to use this formalization to 
represent annotations. Storage in RDF format improves interoperability and sharing data between annotation systems. Therefore, Cabanac et al. [41] indicate that " $R D F$ based annotation can be used by different software if they structure the data in the same way". Besides, some annotation systems enrich the practice of electronic annotation by making of every annotation an entry point for a debate in context. In fact, any learner can respond to an annotation by associating for it an answer; forming thus a discussion thread. Thereafter, responses may also lead to new answers. Thus, each annotation can initiate a forum in context represented by a tree of responses [42].

\section{$F$. To notify and answer to annotations}

Some annotation systems provide to learners the possibility to interact by answering to annotations of the other students. CRAS-RAIDS [23], Tsaap-Notes [11] and Note-Taking [18], among others, offer feature of threads just like forums. Annotations of the same thread are then bound between them in chronological order. With this feature, the users of such systems benefit from annotations in context left by previous annotators [11]. However, each student can comment a resource or an annotation at any time. To inform concerned learners without requiring them to constantly revisit the annotated resource or the added annotation, which they are supervised commented them, notification techniques were used. For example, in the discussions software of Microsoft OneNote, notifications are sent via email and the details of the content are customizable [48].

\section{G. To recommend data related to annotations}

The technique of recommendation of information allows to identify and to propose automatically information considered useful by annotation system for a given learner related to his annotations. For example, a feature designed to help the learner to enrich his knowledge about the studied domain is presented by EyeGaze [5], ASRLM [16], and Crocodoc [46]. These annotation systems identify a list of documents by making inferences from the annotated passages and other documents in database annotations. The items in this list are inserted at the end of document displayed in a section entitled 'to read'. Ferretti et al, [28] present a prototype of annotation system called We-LCoME which determines by means of judgments (sentences), expressed towards the annotated passages, a value of correlation between learners. Thus, students who have similar profiles are mutualized to create an information exchange. Another example of recommendation of data related to annotations is provided by Kalboussi et al, [2, 31] by presenting a new annotation system New-WebAnnot able to recommend web services assistance interpreted from the learner's annotations [53]. Based on a new approach presenting the learner's annotative activity as a means to invoke web services implicitly [38], the proposed annotation system tries to assist the learner via web services during his learning activities [52]. Therefore, from a user's annotation, the system is able to interpret a semantic implicitly expressed which presents a need for a web service to meet annotation goals [34]. Based on this extracted semantic, the annotation system discovers and invokes the requested web service. In the same context, Omheni et al. $[35,36]$ try to extract the personality traits of the learner from his annotations with the aim of recommending a well-defined profile for each annotator. Therefore, Omheni et al. [32] present an approach that can measure some personality traits (conscientiousness and neuroticism) with reasonable accuracy by reference to learner's digital annotation practices [33].

\section{COMPARATIVE STUdy OF FUnCTIONALITIES PROVIDED BY ANNOTATION SYSTEMS}

We expose in table I a comparative study of forty annotation systems developed during the last decade around the seven functionalities presented above. The systems are presented according to the chronological order of their publication or update year (from 2005 until 2015). In the case where the annotation system has several versions, we take the recent version to follow the updates in each annotation system. In addition, we present for each system the consulted reference.

TABLE I.

COMPARATIVE STUdY OF FUNCTIONALITIES PROVIDED BY ANNOTATION SYSTEMS FOR LEARNERS IN EDUCATIONAL CONTEXT

\begin{tabular}{|c|c|c|c|c|c|c|c|c|c|}
\hline \multirow[b]{2}{*}{$\begin{array}{c}\text { Name of } \\
\text { annotation system }\end{array}$} & \multirow[b]{2}{*}{ Year } & \multirow{2}{*}{ 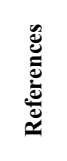 } & \multicolumn{7}{|c|}{ Functionalities } \\
\hline & & & $\begin{array}{c}\text { to manage } \\
\text { annotations } \\
\text { (create/delete/ } \\
\text { modify) }\end{array}$ & $\begin{array}{c}\text { to store } \\
\text { annotations }\end{array}$ & $\begin{array}{l}\text { to visualize } \\
\text { stored } \\
\text { annotations }\end{array}$ & $\begin{array}{c}\text { to search } \\
\text { annotations }\end{array}$ & $\begin{array}{c}\text { to share } \\
\text { annotations }\end{array}$ & $\begin{array}{l}\text { to notify and } \\
\text { answer to } \\
\text { annotations }\end{array}$ & $\begin{array}{c}\text { to recommend } \\
\text { data related to } \\
\text { annotations }\end{array}$ \\
\hline Livenotes & 2005 & [14] & $\checkmark$ & $\checkmark$ & $\checkmark$ & & & & \\
\hline TafAnnote & 2005 & [41] & $\checkmark$ & $\checkmark$ & $\checkmark$ & $\checkmark$ & $\checkmark$ & $\checkmark$ & $\checkmark$ \\
\hline CASE & 2006 & [20] & $\checkmark$ & $\checkmark$ & $\checkmark$ & & $\checkmark$ & & $\checkmark$ \\
\hline Scrapbook & 2006 & [47] & $\checkmark$ & $\checkmark$ & $\checkmark$ & & $\checkmark$ & $\checkmark$ & \\
\hline u-Annotate & 2006 & [9] & $\checkmark$ & $\checkmark$ & $\checkmark$ & & $\checkmark$ & $\checkmark$ & \\
\hline Notebook & 2007 & [26] & $\checkmark$ & $\checkmark$ & $\checkmark$ & $\checkmark$ & & $\checkmark$ & \\
\hline PaperCP & 2007 & [27] & $\checkmark$ & $\checkmark$ & $\checkmark$ & & $\checkmark$ & & \\
\hline A.nnotate & 2008 & [44] & $\checkmark$ & $\checkmark$ & $\checkmark$ & $\checkmark$ & $\checkmark$ & $\checkmark$ & \\
\hline CKB system & 2008 & [45] & $\checkmark$ & $\checkmark$ & $\checkmark$ & & & & $\checkmark$ \\
\hline We-LCoME & 2008 & [28] & $\checkmark$ & $\checkmark$ & $\checkmark$ & & & & \\
\hline CoScribe & 2009 & [10] & $\checkmark$ & $\checkmark$ & $\checkmark$ & & $\checkmark$ & $\checkmark$ & \\
\hline WriteOn 1.0 & 2009 & [13] & $\checkmark$ & $\checkmark$ & $\checkmark$ & $\checkmark$ & & & \\
\hline Crocodoc & 2010 & [46] & $\checkmark$ & $\checkmark$ & $\checkmark$ & $\checkmark$ & $\checkmark$ & & \\
\hline PAMS 2.0 & 2010 & [17] & $\checkmark$ & $\checkmark$ & $\checkmark$ & & $\checkmark$ & $\checkmark$ & \\
\hline Vpen & 2010 & [22] & $\checkmark$ & $\checkmark$ & $\checkmark$ & & $\checkmark$ & & $\checkmark$ \\
\hline WebAnnot-PAML & 2010 & [25] & $\checkmark$ & $\checkmark$ & $\checkmark$ & $\checkmark$ & & & \\
\hline OATS & 2011 & [40] & $\checkmark$ & $\checkmark$ & $\checkmark$ & $\checkmark$ & $\checkmark$ & $\checkmark$ & \\
\hline MobiTOP & 2012 & [7] & $\checkmark$ & $\checkmark$ & $\checkmark$ & & $\checkmark$ & & $\checkmark$ \\
\hline
\end{tabular}


PAPER

FUNCTIONALITIES PROVIDED BY ANNOTATION SYSTEMS FOR LEARNERS IN EDUCATIONAL CONTEXT: AN OVERVIEW

\begin{tabular}{|c|c|c|c|c|c|c|c|c|c|}
\hline \multirow[b]{2}{*}{$\begin{array}{c}\text { Name of } \\
\text { annotation system }\end{array}$} & \multirow[b]{2}{*}{ Year } & \multirow{2}{*}{ 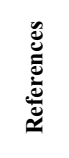 } & \multicolumn{7}{|c|}{ Functionalities } \\
\hline & & & $\begin{array}{c}\text { to manage } \\
\text { annotations } \\
\text { (create/delete/ } \\
\text { modify) }\end{array}$ & $\begin{array}{c}\text { to store } \\
\text { annotations }\end{array}$ & $\begin{array}{l}\text { to visualize } \\
\text { stored } \\
\text { annotations }\end{array}$ & $\begin{array}{c}\text { to search } \\
\text { annotations }\end{array}$ & $\begin{array}{c}\text { to share } \\
\text { annotations }\end{array}$ & $\begin{array}{l}\text { to notify and } \\
\text { answer to } \\
\text { annotations }\end{array}$ & $\begin{array}{l}\text { to recommend } \\
\text { data related to } \\
\text { annotations }\end{array}$ \\
\hline MyNote & 2012 & [6] & $\checkmark$ & $\checkmark$ & $\checkmark$ & & $\checkmark$ & & \\
\hline ASHE & 2013 & [24] & $\checkmark$ & $\checkmark$ & $\checkmark$ & & & & \\
\hline CATool & 2013 & [43] & $\checkmark$ & $\checkmark$ & $\checkmark$ & & & $\checkmark$ & \\
\hline CLAS & 2013 & [1] & $\checkmark$ & $\checkmark$ & $\checkmark$ & $\checkmark$ & $\checkmark$ & & \\
\hline $\begin{array}{l}\text { Microsoft OneNote } \\
\text { (version 15.0) }\end{array}$ & 2013 & [48] & $\checkmark$ & $\checkmark$ & $\checkmark$ & $\checkmark$ & $\checkmark$ & & \\
\hline $\begin{array}{l}\text { Open Annotation } \\
\text { Collaboration }\end{array}$ & 2013 & [42] & $\checkmark$ & $\checkmark$ & $\checkmark$ & & $\checkmark$ & & \\
\hline WebAnnot & 2013 & {$[4]$} & $\checkmark$ & $\checkmark$ & $\checkmark$ & $\checkmark$ & $\checkmark$ & & \\
\hline $\begin{array}{l}\text { Adobe Reader } \\
\text { (version XI) }\end{array}$ & 2014 & [50] & $\checkmark$ & $\checkmark$ & $\checkmark$ & $\checkmark$ & $\checkmark$ & $\checkmark$ & $\checkmark$ \\
\hline ASRLM & 2014 & [16] & $\checkmark$ & $\checkmark$ & $\checkmark$ & & & & \\
\hline EndNotes X7.2 & 2014 & {$[8]$} & $\checkmark$ & $\checkmark$ & $\checkmark$ & $\checkmark$ & $\checkmark$ & $\checkmark$ & \\
\hline Com2Anno-iRuns & 2014 & [12] & $\checkmark$ & $\checkmark$ & $\checkmark$ & & & & \\
\hline CRAS-RAIDS & 2014 & [23] & $\checkmark$ & $\checkmark$ & $\checkmark$ & & & & \\
\hline Eye-Gaze & 2014 & {$[5]$} & $\checkmark$ & $\checkmark$ & $\checkmark$ & $\checkmark$ & $\checkmark$ & & $\checkmark$ \\
\hline IIAF & 2014 & [21] & $\checkmark$ & $\checkmark$ & $\checkmark$ & & & & \\
\hline Note-taking system & 2014 & [18] & $\checkmark$ & $\checkmark$ & $\checkmark$ & & & $\checkmark$ & \\
\hline Tsaap-Notes & 2014 & [11] & $\checkmark$ & $\checkmark$ & $\checkmark$ & & $\checkmark$ & $\checkmark$ & $\checkmark$ \\
\hline VSim & 2014 & [19] & $\checkmark$ & $\checkmark$ & $\checkmark$ & $\checkmark$ & & & \\
\hline New-WebAnnot & 2015 & {$[2]$} & $\checkmark$ & $\checkmark$ & $\checkmark$ & $\checkmark$ & $\checkmark$ & & $\checkmark$ \\
\hline $\begin{array}{l}\text { Online-Annotation- } \\
\text { System }\end{array}$ & 2015 & [15] & $\checkmark$ & $\checkmark$ & $\checkmark$ & & $\checkmark$ & & \\
\hline $\begin{array}{l}\text { PDF-Annotator } \\
\text { (version 5.0.0.505) }\end{array}$ & 2015 & [49] & $\checkmark$ & $\checkmark$ & $\checkmark$ & $\checkmark$ & $\checkmark$ & $\checkmark$ & $\checkmark$ \\
\hline
\end{tabular}

\section{DISCUSSION AND OUTLOOK}

According to the comparative table of functionalities provided by annotation systems presented previously, we note that the majority of annotation tools present the same functionalities for managing annotations (adding, deletion and modification), storing annotations or visualizing them. The presence of these three features into the architecture of all annotation systems shows that these functionalities are required in any practical electronic annotation similar to the pen and paper annotation. On the other hand, and with the emergence of information technology, annotation systems have benefited of this evolution by supplying new features. Thus, the search for annotations according to several criteria or the sharing of annotations between groups of learners appears as new services offered by the developers of annotation tools.

However, few annotation systems present the functionality of recommendation of data to learner interpreted from his annotations. This service requires an analysis of the semantics of annotation to infer knowledge implicitly expressed into the annotative activity of learner. This deducted information is exploited afterward either by others students or by machine. Thus, recent works focus to provide annotation systems able to recommend data interpreted from learner's annotations. We can quote in this context some research works such as: PDF-Annotator [49] that offers PDFs related to the same topic of annotated text; CASE [20] and Vpen [22] which present videos or images related to the semantics of annotations realized by learner; New-WebAnnot [2] that recommends an assistance by web services interpreted implicitly through learner's annotative activity; Tsaap-Notes [11] which provides a learner profile constructed from his social annotations; and Omheni et al. [32] who interpret the personality traits of learner from his annotations.

In another view point, e-lecture systems enable educators to prepare learning material, to enhance it with annotations before, during, or after a presentation, and to present it on a public display or in a lecture recording, respectively. For example, those annotations can be highlighted or underlined text, or provide text notes or sketches [4]. Usually, learners passively consume the learning material and its annotations. In contrast, some of the Web 3.0 key ideas are user generated content as well as an active participation and collaboration of users. These ideas are taken up to apply for e-learning [39]. Current research on Web 3.0 and e-learning also shows the integration of lecture recordings with Web 3.0 applications [3] as well as benefits and opportunities resulting from learner participation and interaction [15]. As participation and active learning are important factors for learning success, it is crucial that students are involved to effectively increase their learning and depth of knowledge [31]. One way to involve students in this process is enabling them to add their own annotations. Annotations can support students in structuring information and to enhance them with their own ideas and knowledge. Interaction can be achieved by exchanging these annotations. The exchange process may have different intentions, for instance sharing knowledge, highlighting or directing attention, asking questions, or collaborating. Sharing annotations may help students to complete or to reflect on their notes. It may even be a stimulus for discussions [39].

On other hand, we can point out that although highly sophisticated annotation systems exist both conceptually as well as technologically, we still observe that their acceptance is somewhat limited on behalf of the learner. Studies made in the works of $[25,31,32]$ show that many readers prefer to print an electronic document and to annotate it on paper instead of annotating it directly on its electronic format using an annotation system. Therefore, the process of marking a paper document with the tools that we find in our environment, a pen, a highlighter, is most preferred by the reader instead of reading a 
PAPER

FUNCTIONALITIES PROVIDED BY ANNOTATION SYSTEMS FOR LEARNERS IN EDUCATIONAL CONTEXT: AN OVERVIEW

document on a screen and the mark via a software interface that requires us to use the keyboard, mouse, stylet, etc. Marshall [54] and Omheni et al. [32] study different kinds of learners annotating paper texts for a variety of reasons. That's because annotation on paper is a seamless and flexible practice. Annotations on electronic texts have generally been more problematic. On some reading platforms, annotation is clunky, interrupting reading as the reader pulls up menus, makes selections, switches to a keyboard to type in text: the learner's attention is refocused on the user interface rather than on the book's content. Electronic annotation tools may also limit a reader's expressive intent (e.g., forcing a highlight to be continuous when the reader wants to fragment it, or imposing neatness on a reader when she wants to scrawl). Sometimes the electronic annotations are stored in infelicitous ways so they are either gone when the learner returns to the eBook on a different computer or so they are recoverable when the reader believes them to be deleted and loans the book or document to a colleague [41].

Also, the aim of the conceptual annotation models is to formalize the main concepts concerning annotations and to define the relationships between annotations and annotated information resources. Therefore, the proposed formal model captures both syntactic and semantic aspects of the annotations [42]. Thus, there are many types of annotation models available in the scientific state of the art and in the already existing end-user applications. We quote in this survey the three main standard frameworks of the annotation: the $\mathrm{W} 3 \mathrm{C}$ annotation project Annotea [29], the IEEE Learning-Object-Metadata (LOM) [30] and the Dublin Core [51]. Using standard formats is preferred, wherever possible, as the investment in marking up resources is considerable and standardization builds in future proofing because new tools, services etc., which were not envisaged when the original semantic annotation was performed may be developed. For annotation systems, standards can provide a bridging mechanism that allows heterogeneous resources to be accessed simultaneously and collaborating users and organizations to share annotations. Because of the need for interoperability, identification and access rights, annotation systems should use basic annotation framework to model the annotation. But, despite the success of RDF technology in the semantic web; the Dublin core in Web technology and IEEE-LOM in the world of learning, few annotation systems adopt these standards to model annotations. This raises a big problem of interoperability and compatibility between these systems since each of them is based on a particular learner annotation model.

Finally, many systems use Tablet PCs and therefore support only two annotation classes: digital ink and text annotations. This may restrict the possibilities of adding information and ideas [21]. Furthermore, the annotation material is usually slides, especially PowerPoint. Only a few systems also facilitate the annotation of other document types or applications [43]. The annotation of annotations is also rarely supported, although it might provide valuable feedback for both educator and students.

Furthermore, many systems focus their use on a certain time period. Those systems, which support only classroom interaction might interrupt the annotation and exchange process and prevent learners to proceed after a lecture. Interviews with students using the Classroom Presenter showed that seeing other students' solutions had a positive effect on their learning [27]. However, many systems allow only the sharing of annotations during a lecture and not afterwards, although the learning process of learners continues after a lecture [9].

Therefore, we think that educational annotation systems should enable both instructors and students to add and exchange annotations during as well as after a lecture. Usually, it is not possible to turn the annotations of the educator on and off, as they stick to the annotation material. This may make it difficult for learners to add their annotations due to less free space. This problem increases if the annotations of other students are displayed as well. In general, this problem is addressed by either reserving extra space for learner annotations or adding several layers. A collaborative filtering approach could be another interesting attempt [11].

The examined systems also do not facilitate to import annotations from another user or to mash up with other services or learning environments. For example, learners often use further learning resources such as forums, Wikis, and Web encyclopedias like Wikipedia in addition to lecture recordings [37]. An integration of such Web (3.0) resources (which can also be seen as annotations in the broader sense) with lecture recordings could help to bring together these different sources and to link them where they semantically belong. By integrating for example a lecture recording with a discussion forum, learners could thereby access a forum discussion directly from a slide that covers the topic the forum posts discuss [11]. Although some systems enable classroom interaction by exchanging student annotations, student participation is limited. No system allows sharing the screen of a learner $\mathrm{PC}$, so that a student can demonstrate his view [15]. This might be an interesting pedagogical scenario offering new teaching and learning scenarios.

\section{CONCLUSION}

We tried to provide in this article a study of some annotation tools used by learners in educational practices. Therefore, we presented a comparison of functionalities provided by forty annotation systems developed in the literature by industry and academia. This comparison involves seven main functionalities provided by annotations tools which are synthesized from the state of the art of all studied systems: to manage annotations; to store annotations; to visualize stored annotations; to search annotations; to share annotations; to notify to annotations and to recommend data related to annotations.

Nevertheless, the outcome of this article has been limited by the inadequate information about annotation systems that were discussed. Some of systems are open source; therefore it is possible to study its documentation and code to explore the structure. However, for many of the other systems, it is very difficult, if not impossible, to get to know their strategies of implementation. Therefore, in the next phase, we will aim to reach a thorough understanding of the implementation and structure of the annotation systems. This tools studied above are necessarily based on annotation models to conceptualize their properties in a formal way to be exploited by computer systems. Thus, we are also planning to propose a survey of conceptual annotation models of learners in digital contents. In another perspective, based on the seven functionalities provided by studied annotation systems, we will try to propose a service of annotation systems 
research. This service presents a user interface providing the possibility of looking for an annotation system which meets the requirements of the learner according to functionalities' annotations tools.

\section{ACKNOWLEDGMENT}

We would like to thank the anonymous reviewers for their extensive comments and suggestions that helped us improve this article.

\section{REFERENCES}

[1] E. Risko, T. Foulsham, S. Dawson, and A. Kingstone, "The Collaborative Lecture Annotation System (CLAS): A New TOOL for Distributed Learning," IEEE Transactions on Learning Technologies, Vol. 6, No. 1, pp.4-13, 2013. http://dx.doi.org/ 10.1109/TLT.2012.15

[2] A. Kalboussi, N. Omheni, O. Mazhoud, and A.H. Kacem, "An Interactive Annotation System to Support the Learner with Web Services Assistance," Proc. of ICALT'15, IEEE, pp.409-410, 2015. http://dx.doi.org/10.1109/ICALT.2015.57

[3] A. Kalboussi, N. Omheni, O. Mazhoud, and A.H. Kacem, "How to Organize the Annotation Systems in Human-Computer Environment: Study, Classification and Observations." Proc. of INTERACT'15, Springer, pp.115-133, 2015. https://dx.doi.org/ 10.1007/978-3-319-22668-2_11

[4] F. Azouaou, H. Mokeddem, L. Berkani, A. Ouadah, and B. Mostefai, "WebAnnot: learner's dedicated web-based annotation tool," International Journal of Technology Enhanced Learning, Vol. 5, No.1, pp.56-84, 2013. http://dx.doi.org/10.1504/IJTEL. 2013.055949

[5] A. Okoso, K. Kunze, and K. Kise, "Implicit gaze based annotations to support second language learning," In Proc. of .UbiComp-Adjunct'14, pp.143-146, http://dx.doi.org/ 10.1145/2638728.2638783

[6] Y.C. Chen, R.H. Hwang, and C.Y. Wang, "Development and evaluation of a Web 2.0 annotation system as a learning tool in an e-learning environment," Computers \& Education, Vol.58, No.4, pp.1094-1105, 2012. http://dx.doi.org/10.1016/j.compedu.2011 .12 .017

[7] D.H. Goh, K. Razikin, C.S. Lee, E.P. Lim, K. Chatterjea, and C.H. Chang, "Evaluating the use of a mobile annotation system for geography education," The Electronic Library, Vol. 30, No.5, pp.589-607, http://dx.doi.org/10.1108/02640471211275666

[8] H. Shibata, and K. Omura, "Effects of Paper on Page Turning: Comparison of Paper and Electronic Media in Reading Documents with Endnotes," In Proc. Of EPC, pp.92-101, 2011. https:// dx.doi.org/10.1007/978-3-642-21741-8 11

[9] M.A. Chatti, T. Sodhi, M. Specht, R. Klamma, and R. Klemke, "u-Annotate: An Application for User-Driven Freeform Digital Ink Annotation of E-Learning Content," In Proc. of ICALT'06, 2006. http://dx.doi.org/10.1109/ ICALT.2006. 1652624

[10] J. Steimle, O. Brdiczka, and M. Muhlhauser, "CoScribe: Integrating Paper and Digital Documents for Collaborative Knowledge Work" IEEE Transactions on Learning Technologies, Vol.2, No.3, 2009. http://dx.doi.org/10.1109/TLT.2009.27

[11] F. Silvestre, P. Vidal, J. Broisin, "Tsaap-Notes -- An Open Microblogging Tool for Collaborative Notetaking during Face-to-Face Lectures," In Proc. of ICALT'14, IEEE, pp.39-43, 2014. http:// dx.doi.org/10.1109/ICALT.2014.22

[12] S.S.J. Lin, H.Y. Chen, Y.T. Chiang, G.H. Luo, and S.M. Yuan, "Supporting Online Reading of Science Expository with iRuns Annotation Strategy," In Proc. of U-MEDIA'14,IEEE, pp. 309312, 2014. http://dx.doi.org/10.1109/U-MEDIA.2014.59

[13] S. Chandrasekar, J. G. Tront, and J. C. Prey, "WriteOn1.0: a tablet PC-based tool for effective classroom instruction," In Proc. Of ITiCSE'09, pp.323-327, 2009. http://dx.doi.org/10.1145/1595496. $\underline{1562975}$

[14] M. Kam, J. Wang, A. Iles, E. Tse, J. Chiu, D. Glaser, O. Tarshish, and J.F. Cannyal., "Livenotes: A System for Cooperative and Augmented Note-Taking in Lectures," In Proc. of CHI'05, pp.531540, 2005. http://dx.doi.org/10.1145/1054972. 1055046
[15] M.H. Chang, R. Kuo, M. Chang, ; Kinshuk, and H.Y. Kung, "Online annotation system and student clustering platform," In Proc. of UMEDIA'15, IEEE, pp. 202-207, 2015. http://dx.doi.org/ 10.1109/UMEDIA.2015.7297455

[16] C.M. Chen, and S.H. Huang "Web-based reading annotation system with an attention-based self-regulated learning mechanism for promoting reading performance," British Journal of Educational Technology, Wiley, Vol.45, No.5, pp. 959-980, 2014. https://dx.doi.org/10.1111/bjet.12119

[17] A.Y. Su, S.J.H. Yang, W.Y. Hwang, and J. Zhang "A Web 2.0based collaborative annotation system for enhancing knowledge sharing in collaborative learning environments,' Computers \& Education, Vol.55, No.2, pp.752-766, 2010. http://dx.doi.org/ 10.1016/j.compedu.2010.03.008

[18] J.K. Kim, W.S. Sohn, K. Hur, and Y.S. Lee, "Increasing learning effect by tag cloud interface with annotation similarity," International Journal of Advanced Media and Communication, Vol.5, No.2-3, pp.135-148, 2014. http://dx.doi.org/10.1504/ IJAMC.2014.060503

[19] L.M. Snyder, "VSim: Scholarly Annotations in Real-Time 3D Environments," In Proc. of DH-CASE '14, Article No. 2, 2014. http://dx. doi.org/10.1145/2657480.2657483

[20] I. Glover, G. Hardaker, Z. Xu, "Collaborative annotation system environment (CASE) for online learning," Campus-Wide Information Systems, Vol.21, No.2, pp.72-80, 2006.http://dx.doi. org/10.1108/10650740410529501

[21] H. Asai, and H. Yamana, "Intelligent Ink Annotation Framework that uses User's Intention in Electronic Document Annotation," In Proc. of ITS'14, pp.333-338, 2014. http://dx.doi.org/10.1145/ $\underline{2669485.2669542}$

[22] W.Y. Hwang, R. Shadiev, S.M. Huang, " Effect of Multimedia Annotation System on Improving English Writing and Speaking Performance," In Proc. Of EDUTAINMENT'10, pp.1-12, 2010. http://dx.doi.org/10.1007/978-3-642-14533-9_1

[23] C.M. Chen, F.Y. Chen, "Enhancing digital reading performance with a collaborative reading annotation system," Computers \& Education, Vol.77, pp.67-81, 2014. http://dx.doi.org/10.1016/ j.compedu.2014.04.010

[24] T.T. Wu, "Using Mobile Annotation System in Public Health Practice Course Based on Project-Based Learning," In Proc. of ICS'12, Springer, pp 349-357, 2012. http://dx.doi.org/ 10.1007/978-3-642-35452-6 36

[25] B. Mostefai, F. Azouaou, A. Balla, "An annotation-based pedagogical memory model for learner," In Proc. of ICMWI'10, pp.90-197, 2010. http://dx.doi.org/10.1109/ICMWI.2010.5647939

[26] J. Rickman, M. Miller, T. Verbick, and K. Todd, "Notebook universities do not have to be expensive," In Proc. of SIGUCCS'06, $\quad$ pp.333-338, 2006. http://dx.doi.org/10.1145/ 1181216.1181287

[27] C. Liao, F. Guimbretière, R. Anderson, N. Linnell, C. Prince, V. Razmov, "PaperCP: Exploring the Integration of Physical and Digital Affordances for Active Learning," In Proc. of INTERACT'07, pp.15-28, http://dx.doi.org/ 10.1007/978-3-540-74800-7_2

[28] S.Ferretti, S. Mirri, L. A. Muratori, M. Roccetti, and P. Salomoni, "E-learning 2.0: you are We-LCoME!," In Proc. Of W4A'08, pp.116-125, http://dx.doi.org/10.1145/1368044. 1368070

[29] J. Kahan, and M.R. Koivunen, "Annotea: an open RDF infrastructure for shared web annotations," In Proc. $W W W^{\prime} 01$, pp.623-632, 2001. http://dx.doi.org/10.1145/371920.372166

[30] IEEE-Standards Association. "IEEE Draft Standard for Learning Object Metadata - Corrigendum 1: Corrigenda for 1484.12.1 LOM (Learning Object Metadata)," IEEE, Piscataway, NJ, pp.1-24, 2010. http://dx.doi.org/10.1109/IEEESTD.2011.5982125

[31] A. Kalboussi, O. Mazhoud, N. Omheni, and A.H. Kacem, "A New Annotation System based on a Semantic Analysis of Learner's Annotative Activity to Invoke Web Services," International Journal of Metadata, Semantics and Ontologies, Vol.9, No.4, pp.350-370, 2014. http://dx.doi.org/10.1504/IJMSO.2014.065447

[32] N. Omheni, A. Kalboussi, O. Mazhoud, and A.H. Kacem, "Automatic Recognition of Personality from Digital Annotations", 
In Proc. of WEBIST'15, pp.273-280, 2015. http://dx.doi.org/ $10.5220 / 0005483002730280$

[33] N. Omheni, A. Kalboussi, O. Mazhoud, and A. Hadjkacem, "Modelling Learner's Personality Profile through Analysis of Annotation Digital Traces in Learning Environment," In Proc. ICALT'15, 2015. http://dx.doi.org/10.1109/ICALT.2015.76

[34] A. Kalboussi, O. Mazhoud, and A.H. Kacem, "Annotative Activity as a Potential Source of Web Service Invocation," In Proc. of WEBIST'13, pp.288-292, 2013. http://dx.doi.org/10.5220/0004369002880292

[35] N. Omheni, O. Mazhoud, A. Kalboussi, A. Hadjkacem, "Prediction of Human Personality Traits from Annotation Activities," In Proc. of WEBIST'14, pp.263-269, 2014. http://dx.doi.org/10.5220/0004801302630269

[36] N. Omheni, O. Mazhoud, A. Kalboussi, A. Hadjkacem, "The Annotation: A Track of Reader's Personality Traits on Paper," In Proc. of ACMSE'14, ACM, article No.10, 2014. http://dx.doi.org/10.1145/2638404.2638469

[37] D. Sulisworo, "Designing the Online Collaborative Learning Using the Wikispaces," International Journal of Emerging Technologies in Learning, Vol.7, No.1, pp.58-61, 2012.

[38] A. Kalboussi, O. Mazhoud, A. Hadj Kacem, and N. Omheni, “A Formal Model of Learner's Annotations Dedicated to Web Services Invocation," In Proc. of ICCE'13, pp.166-169, 2013.

[39] C. Cannata, "Folksonomy, Tagging and Taxonomy for Effective Learning. Perspectives of Learning 2.0 in the XXI century," International Journal of Emerging Technologies in Learning, Vol.4, No.2, pp.26-32, 2009.

[40] S. Bateman, R. Farzan, P. Brusilovsky, and G. McCalla, "OATS: The Open Annotation and Tagging System," In Proceedings of I2LOR, 2011.

[41] G. Cabanac, M. Chevalier, C. Chrisment, and C. Julien, "A Social Validation of Collaborative Annotations on Digital Documents," In Proc. of IWAC'05, pp.31-40, 2005.

[42] R. Sanderson, P. Ciccarese, and H.V de Sompel, "Open Annotation Data Model," W3C Community Draft, 2013.

[43] A. Barret, J. Celevenger, and J. Martini," Open Sourcing Harvard University's Collaborative Annotation Tool," Academic Technology Services, Harvard University, 2013. Available at : http://blogs.law.harvard.edu/acts/files/2012/06/handout.pdf

[44] C. Anagnostopoulou, and F. Howell, "Collaborative online annotation of musical scores for e-Learning using A.nnotate.com," In Proc. of ITED'09, pp.4071-4079, 2009.

[45] B. Settles, M. Craven, and L. Friedland, "Active Learning with Real Annotation Costs," In Proceedings of NIPS'08, 2008.

[46] "Using Crocodoc to Annotate Submissions," Maryland University, 2010. Available at: https://elms.umd.edu/sites/ elms.umd.edu/files/webfiles/documents/Using_Crocodoc.pdf

[47] Ma, F. and Murota, M., "Development of Web Browser Extension for Cross Sectional Search of History, Bookmarks and ScrapBook," IEICE Rep., Vol.106, No.583, pp.93-98, 2007.

[48] P. Weverka, "Microsoft OneNote 2013 Plain \& Simple". Microsoft Press, ACM, 2013.

[49] "PDF Annotator manual," GRAHL software design, Available at: http://www.pdfannotator.com/

[50] Adobe Reader, "Instructions on the annotation of Pdf files," PannotatePdfv11, Elsevier, 2014.
[51] P. Carmichael, "Learning how to learn: using the Dublin Core metadata element set to support teachers as researchers," In Proc. of DCMA'02, pp.201-203, 2002.

[52] A. Kalboussi, O. Mazhoud, N. Omheni, and A.H. Kacem, "An Approach of Assistance of Learner's Annotative Activity through Web Services," Australian Journal of Intelligent Information Processing Systems, Vol.13, No. 3, pp.15-22, 2013. Available at: https://cs.anu.edu.au/ojs/index.php/ajiips/article/view/1243

[53] O. Mazhoud, A. Kalboussi, N. Omheni, and A.H. Kacem, "Enhancing Learner's Activities through Recommendations based on Annotations'. In Proc. of ICCE'15, pp.281-287, 2015.

[54] C.C. Marshall, "Reading and Writing the Electronic Book," Morgan \& Claypool, Chapel Hill, NC, 2010.

[55] Resource Description Framework (RDF), World Wide Web Consortium (W3C), Available at: http://www.w3.org/RDF/

\section{AUTHORS}

Anis Kalboussi received his Master's degree and his $\mathrm{PhD}$ in Computer Science from the Higher Institute of Computer Science and Management of Kairouan and the Faculty of Economics and Management of Sfax in 2011 and 2015, respectively. $\mathrm{He}$ is currently an Associate Professor in Computer Science at the University of Kairouan, Tunisia. He is a member of the ReDCAD Research Laboratory. His current research areas include Technology Enhanced Learning, Semantic Web, Personal Information Management, Web Services, and metadataannotation.

Omar Mazhoud received his Master's degree in Computer Science from UPS, University Paul Sabatier Toulouse-III in 1991. He is currently an Assistant Professor in Computer Science at the University of Kairouan, Tunisia. He is a Member of the ReDCAD Research Laboratory. He is an IEEE Member. Since 2010, he is a PhD student at the University of Sfax. His PhD subject focuses on a new approach guided by web services to predict and satisfy the annotator's reading goal from his annotative activity. Its application domains are metadataannotation, human computer interaction (HCI), and Technology Enhanced Learning.

Ahmed Hadj Kacem received his MS and his $\mathrm{PhD}$ in Computer Science from University Paul Sabatier Toulouse-III in 1991 and 1995, respectively. He joined the University of Sfax, Tunisia as an Assistant Professor in 1997, then as a Full Professor in 2007. Since 2014, he is the Doyen of the Faculty of Economics and Management, Sfax. He is a Member of the ReDCAD Research Laboratory. His current research areas include multi-agent systems and adaptive software architectures for distributed systems. He is an IEEE Senior Member and he is Founder and Chair of the Computer Society Chapter in 2009.

Submitted, 25 October 2015. Published as resubmitted by the authors on 28 January 2016. 